October 31, 2018

\title{
An $a b$ initio relativistic coupled-cluster theory of dipole and quadrupole polarizabilities: Applications to a few alkali atoms and alkaline earth ions
}

\author{
B. K. Sahoo $\dagger$ \\ Max-Planck Institute for the Physics of Complex Systems \\ Nöthnitzer Straße 38, D-01187 Dresden, Germany
}

(Dated: October 31, 2018)

\begin{abstract}
We present a general approach within the relativistic coupled-cluster theory framework to calculate exactly the first order wave functions due to any rank perturbation operators. Using this method, we calculate the static dipole and quadrupole polarizabilities in some alkali atoms and alkaline earthmetal ions. This may be a good test of the present theory for different rank and parity interaction operators. This shows a wide range of applications including precise calculations of both parity and $\mathrm{CP}$ violating amplitudes due to rank zero and rank one weak interaction Hamiltonians. We also give contributions from correlation effects and discuss them in terms of lower order many-body perturbation theory.
\end{abstract}

PACS numbers: 31.15.Ar,31.15.Dv,31.25.Jf,32.10.Dk

For a long time, studies of dipole and quadrupole polarizabilities have been highly interesting in many important aspects for both neutral atoms and ions [1, 2, 3, 4, 5, 6, 7, 8, 9, 10, 11]. These quantities are used in the case of ions to remove the quadratic Stark shifts in the recent proposed optical frequency standards where suitable instruments are not available for very precise measurements [12, 13]. Relativistic effects are also investigated for these properties [14, 15]. Often theoretical studies are limited by many approximations to only approximate accuracy of the results [17, 21]. In many cases, semiempirical methods, which combine the calculated E1 and E2 matrix elements of important states and the experimental excitation energies, are used to calculate these quantities [18, 19]. Core electron contributions are always only estimated in this approach 1,18$]$. Therefore, an $a b$ initio method is necessary to be able to, in principle, explain the importance of the electron correlation effects in the above properties and also test the manybody theories employed. These results are largely used to determine the van der Waals coefficients or dispersion factors [19, 20, 21, 22]. In some cases, the Dirac-Fock (DF) method and relativistic random-phase approximations (RPA) for the Dalgarno-Lewis [21, 22, 23] or scalar relativistic Douglas-Kroll [15, 16] effective Hamiltonians are used to determine these quantities. A number of calculations on these quantities using molecular codes and pseudopotentials have also been reported [9, 15, 16, 17].

Using the relativistic coupled-cluster (RCC) theory, we report here a novel approach for the first time to calculate wave functions up to all orders in the residual Coulomb interaction and first order due to any kind of perturbed operator in one valence systems. This takes care of the sum-over-states approach of the many-body perturbation

\footnotetext{
†E-mail: bijaya@mpipks-dresden.mpg.de
}

theory (MBPT) as an exact solution. This method can be applied to calculate the first order wave function due to dipole and quadrupole transition operators. Hence, polarizabilities can be obtained by calculating the expectation values of the corresponding operators. As an $a b$ initio test of the theory, it is also possible to calculate the light shift ratio in ions like $\mathrm{Ba}^{+}$using the present method as proposed in 24]. Moreover, parity non-conserving (PNC) and CP violating amplitudes can be calculated very precisely using this method due to rank zero or rank one weakly interacting Hamiltonian, which are the most challenging studies during the last three decades [25, 26].

To demonstrate the application of the method, we consider three systems each from alkali atoms ( $\mathrm{Li}, \mathrm{Na}$ and $\mathrm{K})$ and alkaline earth ions $\left(\mathrm{Be}^{+}, \mathrm{Mg}^{+}\right.$and $\left.\mathrm{Ca}^{+}\right)$to calculate their dipole and quadrupole polarizabilities having different angular momentum selection rules.

The energy shift, $\Delta E\left(J_{n}, M_{n}\right)$ of any state $\mid J_{n} M_{n}>$, with principal quantum number $n$, in a direct current (dc) electric field $\overrightarrow{\mathbf{E}}=\mathcal{E} \hat{\mathbf{z}}$ can be expressed as

$$
\Delta E\left(J_{n}, M_{n}\right)=-\frac{1}{2} \alpha^{1}\left(J_{n}, M_{n}\right) \mathcal{E}^{2},
$$

where $\alpha^{1}\left(J_{n}, M_{n}\right)$ is defined as the static polarizability of state $\left|J_{n} M_{n}\right\rangle$. Further, $\alpha^{1}\left(J_{n}, M_{n}\right)$ can be divided as

$\alpha^{1}\left(J_{n}, M_{n}\right)=\alpha_{0}^{1}\left(J_{n}, M_{n}\right)+\frac{3 M_{n}^{2}-J_{n}\left(J_{n}+1\right)}{J_{n}\left(2 J_{n}-1\right)} \alpha_{2}^{1}\left(J_{n}, M_{n}(0.2)\right.$

Here $\alpha_{0}^{1}\left(J_{n}, M_{n}\right)$ and $\alpha_{2}^{1}\left(J_{n}, M_{n}\right)$ are known as the scalar and tensor polarizabilities, respectively. From the first order perturbation equations, these parameters can be expressed as the sum over intermediate states

$$
\alpha_{i}^{1}\left(J_{n}, M_{n}\right)=-2 \sum_{k \neq n} C_{i}^{1} \frac{\left|\left\langle J_{n} M_{n}|z| J_{k} M_{k}\right\rangle\right|^{2}}{E_{n}-E_{k}},
$$

where $i$ represents either 0 or $2, C_{i}^{1}$ are the appropriate angular co-efficients, $z$ is the $\hat{\mathbf{z}}$ component of the position 
vector $\overrightarrow{\mathbf{r}}$ and $E$ 's are the unperturbed energy levels. Since $z$ can be expressed in terms of the spherical harmonics of rank one $\left(Y_{10}(\theta, \phi)\right)$, the above matrix elements will be non-zero between opposite parity states satisfying the E1 transition selection rules. The $\alpha_{i}$ 's can be expressed in terms of the reduced matrix elements of the E1 operator $(D=e \overrightarrow{\mathbf{r}})$ as follows

$$
\alpha_{0}^{1}\left(J_{n}\right)=\frac{-2}{3\left(2 J_{n}+1\right)} \sum_{k \neq n} \frac{\left|\left\langle J_{n}\|D\| J_{k}\right\rangle\right|^{2}}{E_{n}-E_{k}}
$$

and

$$
\begin{array}{r}
\alpha_{2}^{1}\left(J_{n}\right)=\left(\frac{40 J(2 J-1)}{3(2 J+3)(2 J+1)(J+1)}\right)^{1 / 2} \sum_{k \neq n}(-1)^{J_{n}+J_{k}+1} \\
\left\{\begin{array}{ccc}
J_{n} & 1 & J_{k} \\
1 & J_{n} & 2
\end{array}\right\} \frac{\left|\left\langle J_{n}|| D|| J_{k}\right\rangle\right|^{2}}{E_{n}-E_{k}} .
\end{array}
$$

Similarly, the static quadrupole polarizability can be expressed as

$$
\begin{aligned}
\alpha_{0}^{2}\left(J_{n}, M_{n}\right) & =-2 \sum_{k \neq n} C_{0}^{2} \frac{\left|\left\langle J_{n} M_{n}|Q| J_{k} M_{k}\right\rangle\right|^{2}}{E_{n}-E_{k}} \\
& =\frac{-2}{5\left(2 J_{n}+1\right)} \sum_{k \neq n} \frac{\left|\left\langle J_{n}\|Q\| J_{k}\right\rangle\right|^{2}}{E_{n}-E_{k}}
\end{aligned}
$$

where $C_{0}^{2}$ is the corresponding angular factor and $Q=$ $-\frac{e}{2}\left(3 z^{2}-r^{2}\right)$ is the E2 operator which has different selection rules than the E1 operator.

The above expressions for both the polarizabilities can be expressed in a general form as

$$
\begin{aligned}
\alpha\left(J_{n} M_{n}\right) & =\left\langle\Psi_{n}|O| \Psi_{n}\right\rangle \\
& =\left\langle\Psi_{n}^{(0)}|O| \Psi_{n}^{(1)}\right\rangle+\left\langle\Psi_{n}^{(1)}|D| \Psi_{n}^{(0)}\right\rangle \\
& =2\left\langle\Psi_{n}^{(1)}|O| \Psi_{n}^{(0)}\right\rangle,
\end{aligned}
$$

where the exact wave function of the $n$th state can be written in terms of the original atomic wave functions and first order corrections due to the corresponding dipole or quadrupole operators $O(=D$ or $Q)$; i.e.

$$
\left|\Psi_{n}\right\rangle=\left|\Psi_{n}^{(0)}\right\rangle+\left|\Psi_{n}^{(1)}\right\rangle .
$$

Note that the $C$ angular factors from the $\alpha$ 's are absorbed in the first order wave functions.

We show that it is possible to calculate $\alpha\left(J_{n} M_{n}\right)$ 's exactly by calculating both the $\left|\Psi_{n}^{(0)}\right\rangle$ and $\left|\Psi_{n}^{(1)}\right\rangle$ using single many-body theory which can avoid the sum-overstates approach given above. In our approach, we would like to obtain the first order perturbed wave function as a solution to the following equation

$$
\left(\mathrm{H}_{0}^{(\mathrm{DC})}-E_{n}^{(0)}\right)\left|\Psi_{n}^{(1)}\right\rangle=\left(E_{n}^{(1)}-\mathrm{H}_{\mathrm{int}}^{\mathrm{E} 1}\right)\left|\Psi_{n}^{(0)}\right\rangle,
$$

where $\mathrm{H}_{0}^{(\mathrm{DC})}$ and $\mathrm{H}_{\text {int }}$ are the Dirac-Coulomb (DC) and interaction Hamiltonians due to E1 or E2 operators, respectively. The $E_{n}^{(0)}$ and $E_{n}^{(1)}$ are the zeroth and first order energies of the $n$th state, respectively.

In the RCC theory, we express the exact wave function for one valence $(n)$ state of a system as

$$
\left|\Psi_{n}\right\rangle=e^{T}\left\{1+S_{n}\right\}\left|\Phi_{n}\right\rangle,
$$

where $\left|\Phi_{n}\right\rangle$ is the open-shell reference state constructed by appending the valence electron ' $n$ ' orbital to the closed-shell Dirac-Hartree-Fock (DF) wave function $\left(\left|\Phi_{0}\right\rangle\right.$; i.e. $\left.\left|\Phi_{n}\right\rangle=a_{n}^{\dagger}\left|\Phi_{0}\right\rangle\right)$. In the above expression, the coupled-cluster (CC) excitation operators, $T$ excites only the core electrons and $S_{n}$ excites either only the valence electron ' $n$ ' or along with necessary core electrons.

To get both the unperturbed and perturbed wave functions of Eqn. (0.8), we express the $T$ and $S_{n}$ operators by

$$
\begin{aligned}
T & =T^{(0)}+T^{(1)} \\
S_{n} & =S_{n}^{(0)}+S_{n}^{(1)}
\end{aligned}
$$

where $T^{(0)}$ and $S_{n}^{(0)}$ are the CC operators for the DC Hamiltonian and $T^{(1)}$ and $S_{n}^{(1)}$ are the corresponding first order excitation operators due to the interaction Hamiltonian. To calculate $\alpha_{i}\left(J_{n} M_{n}\right)$, only linear terms involv$\operatorname{ing} T^{(1)}$ or $S_{n}^{(1)}$ operators are kept from the exponential function of Eqn. (0.10)

$$
\left|\Psi_{n}\right\rangle=e^{T^{(0)}}\left\{1+\left(1+S_{n}^{(0)}\right) T^{(1)}+S_{n}^{(1)}\right\}\left|\Phi_{n}\right\rangle,(0.13)
$$

Hence, the unperturbed and perturbed wave functions can be separated as

$$
\begin{aligned}
& \left|\Psi_{n}^{(0)}\right\rangle=e^{T^{(0)}}\left\{1+S_{n}^{(0)}\right\}\left|\Phi_{n}\right\rangle \\
& \left|\Psi_{n}^{(1)}\right\rangle=e^{T^{(0)}}\left\{\left(1+S_{n}^{(0)}\right) T^{(1)}+S_{n}^{(1)}\right\}\left|\Phi_{n}\right\rangle .
\end{aligned}
$$

We consider only the single and double excitations from the RCC method (CCSD method) in our calculations, which is proved successful to cope with the electron correlation effects in most one valence systems; i.e.

$$
\begin{aligned}
T & =T_{1}+T_{2} \\
S_{n} & =S_{1 n}+S_{2 n} .
\end{aligned}
$$

First we solve the unperturbed $T^{(0)}$ and $S_{v}^{(0)}$ amplitudes by solving usual CC equations, then these amplitudes are used to determine the $T^{(1)}$ and $S_{v}^{(1)}$ amplitudes in the following equations

$$
\begin{array}{r}
\left\langle\Phi_{0}^{*}\left|\overline{\mathrm{H}_{N}^{(\mathrm{DC})}} T(1)\right| \Phi_{0}\right\rangle=-\left\langle\Phi_{0}^{*}\left|\overline{\mathrm{H}_{\mathrm{int}}}\right| \Phi_{0}\right\rangle \\
\left.\left\langle\Phi_{n}^{*}\right| \overline{\left(\mathrm{H}_{N}^{(\mathrm{DC})}\right.}-\mathrm{IP}_{n}\right) S_{n}^{(1)}\left|\Phi_{n}\right\rangle= \\
-\left\langle\Phi_{n}^{*}\left|\left[\overline{\mathrm{H}_{\mathrm{int}}}\left\{1+S_{n}^{(0)}\right\}+\overline{\mathrm{H}_{N}^{(\mathrm{DC})}} T^{(1)}\right]\right| \Phi_{n}\right\rangle,
\end{array}
$$

where the subscript $N$ represents normal order form of the operators, $\mathrm{IP}_{n}$ is the ionization potential of the $n$th state and the symbol * represents excited states with respect to the corresponding reference states.

After getting both the unperturbed and perturbed amplitudes, we evaluate polarizabilities using the following expression 


$$
\begin{aligned}
\alpha_{i}\left(J_{n} M_{n}\right) & =\frac{\left\langle\Phi_{n}\left|\left\{1+S_{n}^{\dagger}\right\} e^{T^{\dagger}} O e^{T}\left\{1+S_{n}\right\}\right| \Phi_{n}\right\rangle}{\left\langle\Phi_{n}\left|\left\{1+S_{n}^{\dagger}\right\} e^{T^{\dagger}} e^{T}\left\{1+S_{n}\right\}\right| \Phi_{n}\right\rangle} \\
& =\frac{\left\langle\Phi_{n}\left|S_{n}^{(1)^{\dagger}} \overline{\mathrm{O}^{(0)}}\left\{1+S_{n}^{(0)}\right\}+\left\{1+S_{n}^{(0)^{\dagger}}\right\} \overline{\mathrm{O}^{(0)}} S_{n}^{(1)}+\left\{1+S_{n}^{(0)^{\dagger}}\right\}\left(T^{(1)^{\dagger} \overline{\mathrm{O}^{(0)}}}+\overline{\mathrm{O}^{(0)}} T^{(1)}\right)\left\{1+S_{n}^{(0)}\right\}\right| \Phi_{n} \gtrless_{(0.20)}\right.}{1+N_{n}^{(0)}}
\end{aligned}
$$

TABLE I: Static dipole and quadrupole polarizabilities in al-

\begin{tabular}{|c|c|c|c|}
\hline Atoms & Expts & Others & This work \\
\hline \multicolumn{4}{|c|}{ Dipole polarizabilities } \\
\hline \multirow[t]{2}{*}{$\mathrm{Li}$} & $164(3.4)[\overline{28}]$ & $163.73[15], 164.6$ [9] & \multirow[t]{2}{*}{162.29} \\
\hline & $148(13)[29]$ & 165.01 [17], 164.111 [6] & \\
\hline \multirow[t]{2}{*}{$\mathrm{Na}$} & $159.2(3.4)[28]$ & 163.07 [18], 164.89 [15] & \multirow[t]{2}{*}{162.89} \\
\hline & $164.6(11.5)$ [29] & 160.7 [9], 165.88 [17] & \\
\hline \multirow[t]{2}{*}{ K } & $292.8(6.1)[28]$ & 290.1 [18], 289.5 [9] & \multirow[t]{2}{*}{286.01} \\
\hline & $305(21.6)[29]$ & 301.28 [15], 285.23 [17] & \\
\hline \multirow{4}{*}{$\mathrm{Li}$} & Quadrul & ole polarizabilities & \multirow{4}{*}{1421.28} \\
\hline & & $1424(4)[21], 1393$ [9] & \\
\hline & & $1423.266(5)[6], 1424$ [30] & \\
\hline & & $1423[31]$ & \\
\hline \multirow[t]{2}{*}{$\mathrm{Na}$} & & 1885(26) [21], 1796 [9] & \multirow[t]{2}{*}{1899.67} \\
\hline & & 1878 [30], 1879 [31] & \\
\hline \multirow[t]{2}{*}{ K } & & $5000(45)[21], 4703$ [9] & \multirow[t]{2}{*}{4919.71} \\
\hline & & 5000 [30], 5001 [31] & \\
\hline
\end{tabular}
kali atoms: $\mathrm{Li}, \mathrm{Na}$ and $\mathrm{K}$.

where for computational simplicity we define $\overline{\mathrm{O}^{(0)}}=$ $e^{T^{(0)^{\dagger}}} \frac{\mathrm{O} e^{T^{(0)}}}{\mathrm{O}^{(0)}}$ and $N_{n}^{(0)}=S_{n}^{(0)^{\dagger}} e^{T^{(0)^{\dagger}}} e^{T^{(0)}} S_{n}^{(0)}$. We compute $\overline{\mathrm{O}^{(0)}}$ in two steps as effective one-body and two-body terms and substitute in the above equation. We account for contributions from the normalization factor expressed as

$$
\text { Norm. }=\left\langle\Psi_{n}|\mathrm{O}| \Psi_{n}\right\rangle\left\{\frac{1}{1+N_{n}}-1\right\}
$$

The above approach can easily be extended for a very precise calculations of PNC amplitudes [25] and CP violating electric dipole moments (EDMs) in atoms 26] by obtaining the first order wavefunctions due to nuclear spin independent and dependent weak interaction Hamiltonians in the place of dipole and quadrupole operators.

We construct the relativistic single particle orbitals using Gaussian type orbitals (GTOs) and we consider the finite size of the nucleus assuming a Fermi charge distribution as discussed in [27]. One can use length and velocity gauge expressions for E1 and E2 operators to verify

\begin{tabular}{|c|c|c|c|}
\hline Atoms & Expts & Others & This work \\
\hline $\mathrm{Be}^{+}$ & Dipole & $\begin{array}{l}\frac{\text { oolarizabilities }}{24.93[9], 24.63[32]} \\
25.04[33], 16.74[34]\end{array}$ & 24.11 \\
\hline $\mathrm{Mg}^{+}$ & $\begin{array}{l}34.62(26)[4] \\
33.0(5)[35] \\
33.8(8)[36]\end{array}$ & $\begin{array}{l}34.144[4], 38.7[37] \\
33.68[9], 37.2[34] \\
34.0[38], 38.9[39]\end{array}$ & 34.59 \\
\hline $\mathrm{Ca}^{+}$ & $\begin{array}{l}70.89(15)[4] \\
75.3(4)[35] \\
72.5(19)[35]\end{array}$ & $\begin{array}{l}70.872[4], 112.4[37] \\
71.01[9], 87(2)[3] \\
76.9[38], 96.2[34]\end{array}$ & 73.86 \\
\hline $\mathrm{Be}^{+}$ & Quadrup & $\begin{array}{l}\frac{\text { le polarizabilities }}{52.93[9], 55.42}[33] \\
55.71[34], 52.4[2]\end{array}$ & 53.80 \\
\hline $\mathrm{Mg}^{+}$ & & $\begin{array}{l}150.2[9], 187.66[34] \\
150.15[38]\end{array}$ & 156.17 \\
\hline $\mathrm{Ca}^{+}$ & & $\begin{array}{l}1171[9], 727.55[34] \\
1303.51[38]\end{array}$ & 706.59 \\
\hline
\end{tabular}

TABLE II: Static dipole and quadrupole polarizabilities in alkaline earth-metal ions: $\mathrm{Be}^{+}, \mathrm{Mg}^{+}$and $\mathrm{Ca}^{+}$.

accuracy of the results. However, we have used only the length gauge expressions which are more stable than others in our calculations. We present our results in Tables II and II, and compare them with the experimental results and other calculated values. Numerous calculations are available for the dipole polarizabilities in the neutral systems. However, we present only a few recent calculations in these tables. To our knowledge, there are only a few experimental results for the dipole polarizabilities whereas no results are found for the quadrupole polarizabilities, though there exist a number of calculations.

We present the DF and different RCC results in Table III to understand the role of electron correlation effects. As seen from this table, $O S_{1 n}^{(1)}$ and its complex conjugate $(c c)$ terms contribute predominantly. To interpret these contributions, we break down this RCC term into some of the leading order MBPT diagrams as shown in the Fig. $\mathbb{1}(\mathrm{a}))$. It is obvious from this relationship that the above term consists of dominant DF (Fig 1 (ai)), core- 
TABLE III: Contributions from DF and important perturbed $\mathrm{CC}$ terms for the dipole and quadrupole polarizabilities.

\begin{tabular}{|c|c|c|c|c|c|}
\hline Atoms & DF & $\bar{O} S_{1 n}^{(1)}+c c$ & $\bar{O} S_{2 n}^{(1)}+c c$ & Norm. & Others \\
\hline \multicolumn{6}{|c|}{ Dipole polarizabilities } \\
\hline $\mathrm{Li}$ & $168 . \overline{95}$ & 164.21 & -0.40 & -0.08 & -1.44 \\
\hline $\mathrm{Na}$ & 188.17 & 169.09 & -1.26 & -0.29 & -4.65 \\
\hline $\mathrm{K}$ & 398.15 & 313.32 & -6.93 & -2.11 & -18.28 \\
\hline $\mathrm{Be}^{+}$ & 24.81 & 24.33 & -0.08 & -0.01 & -0.13 \\
\hline $\mathrm{Mg}^{+}$ & 38.39 & 35.57 & -0.43 & -0.04 & -0.51 \\
\hline $\mathrm{Ca}^{+}$ & 94.62 & 79.84 & -1.48 & -0.32 & -4.18 \\
\hline \multicolumn{6}{|c|}{ Quadrupole polarizabilities } \\
\hline $\mathrm{Li}$ & $14 \overline{84.98}$ & 1444.87 & 0.00 & -0.72 & -22.87 \\
\hline $\mathrm{Na}$ & 2230.62 & 2012.95 & 0.00 & -3.34 & -109.94 \\
\hline K & 7099.70 & 5562.48 & 0.00 & -36.38 & -606.39 \\
\hline $\mathrm{Be}^{+}$ & 54.97 & 54.23 & 0.00 & -0.02 & -0.41 \\
\hline $\mathrm{Mg}^{+}$ & 171.34 & 161.09 & 0.00 & -0.18 & -4.74 \\
\hline $\mathrm{Ca}^{+}$ & 952.65 & 750.39 & 0.00 & -3.07 & -40.73 \\
\hline
\end{tabular}

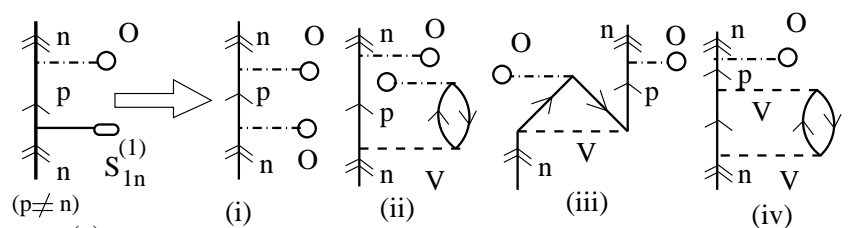

(a)

(i)

(ii)

(iv)

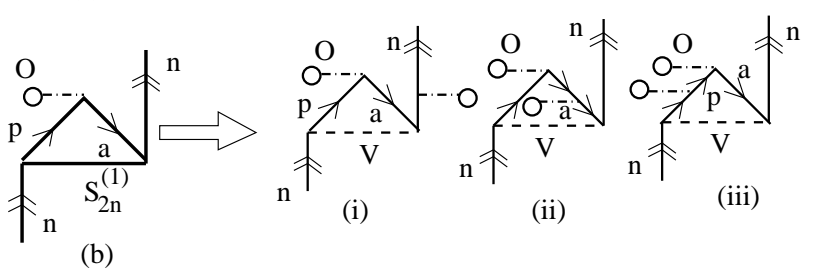

FIG. 1: Break down of important perturbed CC diagrams into some of the important lowest order MBPT diagrams. The $V$ represents Coulomb operator and a and p represent, occupied and unoccupied orbitals, respectively.

polarization (Fig 1(aii) and 1(aiii)) and pair-correlation (Fig1(aiv)) effects, therefore this results in large contributions for all the systems. However, we also show in the Fig. (1)(b)) another important RCC term $\left(O S_{2 n}^{(1)}\right)$ as the sum of different types of core-polarization effects. From Table III] we find that contributions from other higher order terms are non-negligible while going towards the large systems. We also notice from this table that the trend of the correlation effects in neutral and ionic systems with the same electronic configurations are different.

In conclusion, we have developed for the first time a general method in the relativistic coupled-cluster theory to calculate the first order wave functions due to any rank operators for both the parities. This technique can be applied to diverse areas of physics ranging from polarizabilities to probe physics beyond the Standard Model of particle physics. We also investigated electron correlation effects from the dipole and quadrupole polarizabilities calculations in six different systems and highlight their behavior. We observed that higher order correlation effects become important in large systems; suggesting that the method can be employed rigorously in heavy systems to obtain accurate results, and can be studied as ab initio tests. Indeed, this approach can also be extended to determine frequency dependent (dynamic) polarizabilities which we defer to our next studies. Conclusively, the many-body aspects presented here would certainly be of interesting for both physicists and quantum chemists.

We are grateful to Professor B. P. Das for many useful discussions and suggesting us to carry out this work. We are also delighted to thank Professor P. Fulde for very enlightening discussions and hospitalities at MPI-PKS.

[1] R. M. Sternheimer, Phys. Rev. A 1, 321 (1970)

[2] L. J. Curtis, Phys. Rev. A 23, 362 (1981)

[3] A. G. Vaidyanathan et al., Phys. Rev. A 26, 3346 (1982)

[4] C. E. Theodosiou, L. J. Curtis and C. A. Nicolaides, Phys. Rev. A 52, 3677 (1995)

[5] M. A. Castro, O. A. V. Amaral and R. Medeiros, Phys. Rev. A 53, 3664 (1996)

[6] Z. C. Yan, J. F. Babb, A. Dalgarno and G. F. W. Drake, Phys. Rev. A 54, 2824 (1996)

[7] V. A. Dzuba, V. V. Flambaum and O. P. Sushkov, Phys. Rev. A 56, R4357 (1997)

[8] J. Zeng, J. Yuan and Q. Lu, Phys. Rev. A 62, 022713 (1998)

[9] S. H. Patil and K. T. Tang, J. Chem. Phys. 106, 2298 (1997); Chem. Phys. Letts. 301, 64 (1999)

[10] E. L. Snow et al, Phys. Rev. A 71, 022510 (2005)

[11] M. W. J. Bromley and J. Mitroy, (in press PRL) (physics/0610045) (2006)

[12] W. M. Itano, J. Res. NIST 105, 829 (2000)

[13] J. M. Amini and H. Gould, Phys. Rev. Letts. 91, 153001 (2003)

[14] E. S. Shuman and T. F. Gallagher, Phys. Rev. A 74, 022502 (2006)

[15] I. S. Lim et al, Phys. Rev. A 60, 2822 (1999)

[16] I. S. Lim et al, J. Chem. Phys. 122, 104103 (2005)

[17] T. Kobayashi, K. Sasagane and K. Y. Chi, Int. J. Quant. Chem. 65, 665 (1997)

[18] M. S. Safronova, W. R. Johnson and A. Derevianko, Phys. Rev. A 60, 4476 (1999)

[19] A. Derevianko et al, Phys. Rev. Lett. 82, 3589 (1999)

[20] X. Chu, A. Dalgarno and G. C. Groenenboom, Phys. Rev. A 72, 032703 (2005)

[21] S. G. Porsev and A. Derevianko, J. Chem. Phys. 119, 844 (2003)

[22] C. Zhu, A. Dalgarno, S. G. Porsev and A. Derevianko, Phys. Rev. A 70, 032722 (2004)

[23] A. Dalgarno and J. T. Lewis, Proc. R. Soc. London 233, $70(1955)$

[24] J. A. Sherman et al., Phys Rev Lett 94, 243001 (2005)

[25] C. S. Wood et al., Science 275, 1759 (1997)

[26] A. Shukla, B. P. Das and J. Andriessen, Phys. Rev. A 
50, 1155 (1994)

[27] R. K. Chaudhuri, P. K. Panda and B. P. Das, Phys. Rev. A 59, 1187 (1999)

[28] R. W. Molof et al, Phys. Rev. A 10, 1131 (1974)

[29] W. D. Hall and J. C. Zorn, Phys. Rev. A 10, 1141 (1974); G. E. Chamberlain, Phys. Rev. A 129, 677 (1963)

[30] M. Marinescu, H. R. Sadeghpour and A. Dalgarno, Phys. Rev. A 49, 982 (1994)

[31] D. Spelsberg, T. Lorenz and W. Meyer, J. Chem. Phys. 99, 7845 (1993)

[32] M. R. Flannery and A. L. Stewart, Proc. Soc. 82, 188 (1963)

[33] P. K. Mukherjee, R. K. Moitra and A. Mukherji, Int. J. Quant. Chem. V, 637 (1971)
[34] P. W. Langhoff and R. P. Hurst, Phys. Rev. 139, A1415 (1965)

[35] E. S. Chang and R. W. Nyoes, Astrophys. J. 269, L61 (1983); E. S. Chang, J. Phys. B 16, L539 (1983)

[36] B. J. Lyons and T. F. Gallagher, Phys. Rev. A 57, 2426 (1998)

[37] S. I. Easa and G. C. Shukla, J. Phys.(Paris) 40, 137 (1979)

[38] S. A. Adelman and A. Szabo, J. Chem. Phys. 58, 687 (1973)

[39] B. Kundu, D. Ray and P. K. Mukherjee, Phys. Rev. A 34, 62 (1982) 